\title{
Avaliação dos níveis de autoestima de mulheres em privação de liberdade
}

\author{
Evaluation of women's self-esteem levels in freedom privacy \\ Patricia Pinheiro Cabral1, \\ Paula Santana Marra ${ }^{1}$, \\ Cecília Amorim de Santana Mota ${ }^{1}$, \\ Juliana Chaves de Mendonça ${ }^{1}$, \\ Antonio Márcio Teodoro Cordeiro Silva ${ }^{2}$, \\ Rogério José de Almeida ${ }^{3}$
}

\begin{abstract}
RESUMO
Este artigo teve por objetivo mensurar os fatores associados aos níveis de autoestima em mulheres em privação de liberdade. Identificou que as mulheres estudadas apresentam em sua maioria, níveis elevados de autoestima. Os principais fatores foram: a prática de alguma religião, receber visitas dos filhos, ter cuidados pessoais, ser vaidosa, se considerar bonita e estar satisfeita com o próprio corpo.
\end{abstract}

PALAVRAS-CHAVE: Autoestima. Privação de liberdade. Saúde mental.

\begin{abstract}
This article aimed to measure the factors associated with levels of self-esteem in women deprived of freedom. He identified that the women studied have mostly high levels of self-esteem. The main factors were: the practice of some religion, receiving visits from children, having personal care, being vain, considering yourself beautiful and being satisfied with your own body.

Keywords: Self-esteem. Deprivation of liberty. Mental Health.
\end{abstract}

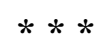

\section{Introdução}

Pessoa privada de liberdade no sistema prisional é todo indivíduo maior de 18 anos custodiado em unidades prisionais, não sendo incluídos os tutelados pelo Sistema

\footnotetext{
${ }^{1}$ Médica, graduada pela Pontifícia Universidade Católica de Goiás (PUC Goiás)

2 Doutor em Biologia Molecular. Professor do Curso de Medicina e do Programa de Pós-Graduação em Ciências Ambientais e Saúde (PPGCAS) da Pontifícia Universidade Católica de Goiás (PUC Goiás).

${ }^{3}$ Doutor em Sociologia. Professor do Curso de Medicina e do Programa de Pós-Graduação em Ciências Ambientais e Saúde (PPGCAS) da Pontifícia Universidade Católica de Goiás (PUC Goiás).
} 
Nacional Socioeducativo (SINASE). Essas pessoas, embora tenham perdido seu direito de ir e vir, ainda conservam seus demais direitos fundamentais. O direito à saúde é garantido tanto pela Constituição Federal em seu artigo 196, que define a saúde como direito de todos e dever do Estado, quanto pelo Sistema Único de Saúde (SUS) (BRASIL, 1988; BRASIL, 2014).

No Levantamento Nacional de Informações Penitenciárias de Mulheres (INFOPEN), publicado em 2018, cujos dados são referentes a informações colhidas até 30 de junho de 2016 em 1418 unidades prisionais, identificou-se que a população prisional feminina no Brasil era de 42.355, sendo que o número de vagas para mulheres era de 27.029. Isso caracteriza uma taxa de ocupação de 156,7\% e um déficit de 15.326 vagas (BRASIL, 2018).

No contexto internacional, o Brasil é o quarto país em número absoluto de mulheres em privação de liberdade, ficando atrás apenas dos Estados Unidos, da China e da Rússia. Em relação ao estado de Goiás, nessa mesma data havia um total de 842 mulheres em privação de liberdade, contra um total de 16.075 homens, figurando como o $12^{\circ}$ estado com a maior população carcerária feminina (BRASIL, 2018).

Em 2014, foi instituída a Política Nacional de Atenção Integral a Saúde das Pessoas Privadas de Liberdade no Sistema Prisional (PNAISP), a qual visa oferecer ações de promoção de saúde e prevenção de agravos no sistema prisional, englobando todo o itinerário carcerário, com o objetivo de garantir o cuidado integral no SUS (BRASIL, 2014). Sabe-se que os serviços de saúde em unidades prisionais são deficientes e que em muitos casos não há a presença de uma equipe multiprofissional, que deveria proporcionar essa assistência à saúde de forma eficaz e com qualidade (SOUZA; CABRAL; SALGUEIRO, 2018).

A condição de saúde das mulheres em privação de liberdade acaba sendo inferior à da população feminina sem essa restrição (BRASIL, 2014). A inadequação dos serviços de saúde está relacionada com a diminuição da saúde física e da saúde mental durante o encarceramento, ou seja, as mulheres não só são mais acometidas por problemas de saúde, como, também, têm um menor acesso aos cuidados de saúde (ALMEIDA et al., 2015).

Um dos fatores que fazem parte da construção da saúde mental é a autoestima. Essa pode ser definida como uma avaliação que um indivíduo faz de si mesmo, envolvendo pensamentos e sentimentos de autoconceito com a aplicação de um 
autovalor. Possui um componente temporal, que pode sofrer variações ao longo da vida, baseado em julgamentos eleitos pelo próprio indivíduo como positivos e negativos, compondo, respectivamente, a auto aprovação e a depreciação (SCHULTHEISZ; APRILE, 2013).

Níveis elevados de autoestima possuem estreita relação com a satisfação com a vida, com a saúde mental, com as habilidades sociais e com o bem-estar. Por outro lado, baixos níveis podem ser associados à incapacidade, à delinquência, à depressão, à ansiedade social, aos transtornos alimentares e à ideação suicida (SCHULTHEISZ; APRILE, 2013).

Dito isto, percebe-se a extrema importância de uma análise da autoestima dessas mulheres, enquanto submetidas ao cárcere, visto que se encontram em situação de extrema vulnerabilidade, de desconstrução de seu próprio conceito de identidade e autoestima, conferindo uma perturbação de seu status quo de saúde mental. A autoestima, além de sua conotação individual, influencia na interação social das mulheres em conjunto com a autoimagem corporal (SILVA, 2013).

Assim, é fato que a saúde mental é negligenciada por parte de instituições de segurança pública. No contexto dos presídios, as internas se encontram em situação de elevada vulnerabilidade para distúrbios mentais, seja por seu contexto anterior à prisão, seja pela própria condição de restrição de liberdade. Nesse sentido, o presente estudo teve por objetivo mensurar os fatores associados aos níveis de autoestima em mulheres em privação de liberdade.

\section{Metodologia}

Trata-se de um estudo transversal analítico com abordagem quantitativa. Esse é um método de pesquisa que busca associar uma exposição com uma doença ou com uma condição de saúde. Nos estudos transversais, a exposição e a condição de saúde/doença são mensuradas de forma concomitante (BASTOS; DUQUIA, 2007). De uma formal geral, nesse tipo de estudo é realizada uma investigação para estabelecer como os fatores citados vão se relacionar com a saúde dentro de uma determinada população (ROTHMAN; GREENLAND; LASH, 2011). 
A pesquisa foi realizada por meio de questionários, aplicados em mulheres apenadas em privação de liberdade na Penitenciária Feminina Consuelo Nasser, localizada no município de Aparecida de Goiânia, Goiás, Brasil.

Como critérios de inclusão, foram pesquisadas mulheres que estavam cumprindo pena em regime fechado e que não estavam em crise de abstinência por algum tipo de droga, lícitas ou ilícitas. O critério de exclusão adotado foi no caso em que as presidiárias não respondessem a todos os itens do questionário.

Foram utilizados dois questionários. Um foi o questionário sociodemográfico criado pelos autores, seguindo a literatura científica. Foram inseridas as seguintes variáveis: idade, etnia, estado civil, religião, atividade física/atividades de recreação, filhos, recebe visitas dos filhos, anos cumpridos na prisão, anos da pena completa, cônjuge/companheiro(a), recebe visitas íntimas, recebe auxilio familiar, realiza trabalho, escolaridade, realiza cuidados pessoais, se considera vaidosa, se considera bonita, está satisfeita com o próprio corpo.

O outro instrumento foi a Escala de Autoestima de Rosenberg (EAR), desenvolvida em 1965, por Morris Rosenberg e aplicado em universitários e idosos na cidade de Nova Iorque. Nessa escala, considera-se que a autoestima é a autoavaliação pessoal, a qual implica sentimento de valor, com componentes afetivos, manifestos de maneira de aprovação ou desaprovação em relação a si mesmo.

A escala foi primeiramente adaptada e validada para a versão Brasileira em 2000, para uso no contexto de cirurgias plásticas (DINI; QUARESMA; FERREIRA, 2004). Em 2007, Avanci realizou a adaptação para uso em adolescentes (AVANCI et al., 2007). Em 2013, Meurer a executou e validou na faixa etária idosa (MEURER et al., 2012a). Sua utilização vem sendo realizada em larga escala no Brasil, aplicada em diversas populações, como gestantes (DIAS et al., 2008; MAÇOLA; DO VALE; CARMONA, 2009), policiais (ANDRADE; SOUSA; MINAYO, 2009) e idosos (MEURER et al., 2012b).

A escala é composta por 10 questões, com respostas dispostas no formato Likert, sendo as duas primeiras positivas e as duas últimas negativas (VISCARDI; CORREIA, 2017). As respostas são: concordo totalmente $=1$; concordo $=2$; discordo $=3$; discordo totalmente $=4$. Dessa maneira, a somatória dos 10 itens compõe o escore da escala, cuja pontuação total oscila de 10 a 40, sendo que a obtenção de uma pontuação alta reflete autoestima elevada. Assim, a autoestima satisfatória ocorre quando o escore é maior ou igual a 30 na referida escala (VISCARDI; CORREIA, 2017). 
Com os dados coletados foi confeccionado um banco de dados utilizando o software IBM SPSS Statistics 18. Foi realizada estatística descritiva com o cálculo de médias, desvio padrão e cálculo das frequências absoluta e relativa percentual. Foi aplicado o teste de normalidade (Kolmogorov-Smirnov) para distinguir as distribuições paramétricas e não-paramétricas. Assim, foram utilizados os testes de Student e ANOVA, bem como os testes Mann-Whitney e Kruskal-Wallis. Para todos os testes comparativos foi assumido p-valor menor ou igual a 0,05 como significativo.

A pesquisa seguiu todos os preceitos legais quanto a ética em pesquisa com seres humanos. O projeto foi aprovado pelo Comitê de Ética e Pesquisa (CEP) da Pontifícia Universidade Católica de Goiás (PUC Goiás) com o parecer n. 3.056.050

\section{Resultados}

Foram entrevistadas 45 mulheres em privação de liberdade, das quais $57,8 \%$ tinham até 35 anos de idade, sendo a média 34,6 anos. A maior idade 55 anos e a menor 20 anos. Dentre as entrevistadas, $62,2 \%$ eram casadas, $73,3 \%$ afirmaram possuir uma religião e $82,2 \%$ possuíam filhos. Dentre elas, $51,1 \%$ cursaram o ensino fundamental e $37,8 \%$ o ensino médio (Tabela 1 ).

Tabela 1. Caracterização sociodemográfica de mulheres em privação de liberdade em presídio de Goiânia, Goiás, Brasil, 2019.

\begin{tabular}{lcc}
\hline Variáveis $(\boldsymbol{n}=\mathbf{4 5})$ & $\boldsymbol{N}$ & $\boldsymbol{f ( \% )}$ \\
\hline Idade (anos) & & 57,8 \\
$\quad$ Até 35 & 26 & 42,2 \\
Acima de 35 & 19 & \\
Etnia & & 20,0 \\
$\quad$ Branca & 9 & 20,0 \\
Negra & 9 & 53,3 \\
Parda & 24 & 6,7 \\
Amarela & 3 & 37,8 \\
Estado Civil & & 62,2 \\
Solteiro & 17 & 73,3 \\
Casado & 28 & 26,7 \\
Religião & & \\
Sim & 33 & 82,2 \\
Não & 12 & \\
Tem Filhos & & \\
Sim & 37 &
\end{tabular}


Você Trabalha

Interno

Externo

Não

Escolaridade

Sabe ler e escrever

Fundamental

Médio

Superior

Caracterizando os aspectos pessoais das entrevistadas, são em sua maioria mulheres que não procuram realizar atividades físicas $(75,6 \%)$ e não recebem visitas íntimas (62,2\%), recebendo, entretanto, visitas dos filhos (62,2\%). Sessenta por cento das entrevistadas deverão cumprir uma pena de até 10 anos. Dentre elas, 82,2\% se consideravam vaidosas, $77,8 \%$ se consideravam bonitas e $88,9 \%$ realizavam cuidados pessoais (Tabela 2 ).

Tabela 2. Caracterização de aspectos pessoais de mulheres em privação de liberdade em presídio de Goiânia, Goiás, Brasil, 2019.

\begin{tabular}{lcc}
\hline Variáveis (n=45) & $\boldsymbol{N}$ & $\boldsymbol{f}(\boldsymbol{\%})$ \\
\hline Recebe Visita Íntima & 17 & 37,8 \\
Sim & 28 & 62,2 \\
Não & & \\
Faz Atividade Física & 11 & 24,4 \\
$\quad$ Sim & 34 & 75,6 \\
Não & & \\
Recebe Visita dos Filhos & 23 & 62,2 \\
Sim & 14 & 37,8 \\
Não & & \\
Tempo de Prisão (anos) & 33 & 73,3 \\
Até 4 & 12 & 26,7 \\
Acima de 4 & & 60,0 \\
Tempo da Pena (anos) & 27 & 40,0 \\
Até 10 & 18 & \\
Acima de 10 & & 37,8 \\
Recebe Auxílio da & 17 & 62,2 \\
Família & 28 & 88,9 \\
Sim & & 11,1 \\
Não & 40 & \\
Tem Cuidados Pessoais & 5 & \\
Sim & & \\
Não & & \\
Você é Vaidosa & & \\
& &
\end{tabular}


Se Considera Bonita

Sim

Satisfeita com seu

Corpo

Sim

Analisando os níveis de autoestima das entrevistadas por meio da Escala de Rosenberg, uma participante foi excluída, por preenchimento incompleto do questionário. Das entrevistadas, $56,8 \%$ pontuaram maior ou igual à 30 pontos, caracterizando uma elevada autoestima, enquanto $43,2 \%$ possuíam baixa autoestima (Tabela 3).

Tabela 3. Análise dos níveis de autoestima de mulheres em privação de liberdade em presídio de Goiânia, Goiás, Brasil, 2019.

\begin{tabular}{lll}
\hline Variáveis $(\mathbf{n}=\mathbf{4 4}) *$ & $\boldsymbol{n}$ & $\boldsymbol{f ( \% )}$ \\
\hline Níveis de Autoestima & & \\
$<30$ (baixa autoestima) & 19 & 43,2 \\
$\geq 30$ (elevada autoestima) & 25 & 56,8 \\
\hline
\end{tabular}

* Uma participante foi excluída por preenchimento incompleto da escala.

No que se refere a comparação dos níveis de autoestima com os dados sociodemográficos foi identificado que as mulheres que afirmara ter religião apresentaram escore mais elevado de autoestima quando comparado com aquelas sem religião $(\mathrm{p}=0,0370)$ (Tabela 4$)$.

Tabela 4. Comparação dos níveis de autoestima com os dados sociodemográficos de mulheres em privação de liberdade em presídio de Goiânia, Goiás, Brasil, 2019.

\begin{tabular}{lccc}
\hline Variáveis $(\boldsymbol{n = 4 4 ) *}$ & Média & DP & p-valor \\
\hline Idade (anos) & & & \\
$\quad$ Até 35 & 28,8 & 5,7 & 0,3125 \\
Acima de 35 & 30,8 & 7,8 & \\
Etnia & & & \\
$\quad$ Branca & 25,7 & 8,1 & \\
$\quad$ Negra & 28,9 & 7,0
\end{tabular}




\begin{tabular}{lccc} 
Parda & 31,3 & 5,9 & 0,1778 \\
Amarela & 31,7 & 4,0 & \\
Estado Civil & & & 0,1416 \\
$\quad$ Solteiro & 27,7 & 7,6 & \\
Casado & 30,8 & 6,0 & $\mathbf{0 , 0 3 7 0}$ \\
Religião & & & \\
$\quad$ Sim & 30,9 & 6,2 & 0,2410 \\
Não & 26,3 & 7,1 & \\
Tem Filhos & & & \\
Sim & 27,1 & 7,9 & 0,9374 \\
Não & 30,2 & 6,4 & \\
Você Trabalha & & & \\
Interno & 29,2 & 8,6 & \\
Externo & 30,0 & 5,8 & \\
$\quad$ Não & 29,4 & 6,7 & \\
Escolaridade & & & \\
$\quad$ Sabe Ler e Fundamental & 28,9 & 6,1 & \\
Médio & 30,0 & 7,0 & \\
Superior & 33,0 & 9,6 & \\
\hline
\end{tabular}

* Uma participante foi excluída por preenchimento incompleto da escala.

* Testes: Teste de t de Student e ANOVA

Já em relação com a comparação entre autoestima e aspectos pessoais foi encontrado que aquelas mulheres que recebiam visita dos filhos $(p=0,0368)$, que tinham cuidados pessoais $(p=0,0163)$, que eram vaidosas $(p=0,0496)$, que se consideravam bonitas $(p=0,0019)$ e que estavam satisfeitas com o seu corpo $(p=0,0396)$ apresentaram valores mais altos de autoestima na Escala de Rosenberg (Tabela 5).

Tabela 5. Comparação dos níveis de autoestima com os aspectos pessoais de mulheres em privação de liberdade em presídio de Goiânia, Goiás, Brasil, 2019.

\begin{tabular}{lccc}
\hline Variáveis $(\boldsymbol{n}=\mathbf{4 4}) *$ & Média & DP & p-valor \\
\hline Recebe Visita Íntima & & & \\
$\quad$ Sim & 30,5 & 6,4 & 0,5299 \\
$\quad$ Não & 29,1 & 6,9 & \\
Faz Atividade Física & & & \\
$\quad$ Sim & 30,0 & 5,6 & 0,8481 \\
$\quad$ Não & 29,5 & 7,1 & \\
Recebe Visita dos Filhos & & & \\
$\quad$ Sim & 31,7 & 6,3 & $\mathbf{0 , 0 3 6 8}$ \\
$\quad$ Não & 27,7 & 5,9 & \\
Tempo de Prisão (anos) & & & \\
Até 4 & 29,3 & 7,1 & 0,5810 \\
Acima de 4 & 30,6 & 5,8 &
\end{tabular}

Tempo de Pena (anos) 
Até 10

Acima de 10

Recebe Auxílio da Família

Sim

Não

Tem Cuidados Pessoais

Sim

Não

Você é Vaidosa

Sim

Não

Se Considera Bonita

Sim

Não

Satisfeita com seu Corpo

Sim

Não

* Uma participante foi excluída por preenchimento incompleto da escala.

* Testes: Teste de $t$ de Student e ANOVA
$29,8 \quad 7,0$

$29,4 \quad 6,3$

$30,4 \quad 6,8$

$29,2 \quad 6,7$

0,5661

$30,5 \quad 5,9$

$23,0 \quad 9,4$

0,0163

$30,4 \quad 6,0$

$26,1 \quad 9,0$

0,0496

$31,3 \quad 5,6$

$24,1 \quad 7,3$

0,0019

$31,1 \quad 5,2$

$26,6 \quad 8,6 \quad \mathbf{0 , 0 3 9 6}$

26,6

\section{Discussão}

O presente estudo permitiu caracterizar o padrão sociodemográfico, os aspectos pessoais, os níveis de autoestima, bem como os fatores associados à maiores ou menores escores de autoestima de mulheres em privação de liberdade na Penitenciária Feminina Consuelo Nasser. Foi possível fazer uma comparação entre as informações obtidas, de forma a identificar quais fatores têm maior influência na autoestima desse grupo de presas.

No presente estudo foi identificado um perfil sociodemográfico com uma idade média de 34,6 anos, se consideram pardas, têm ensino fundamental completo, são casadas e que em sua grande maioria têm filhos e recebem visitas desses filhos. Já em relação ao tempo de pena, a maioria foi de até 10 anos.

Comparado com um estudo que apresentou dados especificamente do Brasil, as mulheres em privação de liberdade tiveram perfil entre 18 e 29 anos (50\%), negras (62\%), têm ensino fundamental incompleto (45\%), solteiras (62\%), com filhos (74\%), tempo médio de pena de 4 a 8 anos e estão encarceradas devido ao tráfico (BRASIL, 2018). O que diverge em parte do padrão que foi encontrado neste estudo.

Já quando comparado com dados de um estudo realizado no Mato Grosso com 56 detentas, os resultados foram mais semelhantes. Esse estudo apresentou uma idade 
média de 32,14 anos. Com mulheres pardas (73,2\%), apenas com ensino fundamental completo $(51,7 \%)$, solteiras $(48,2 \%)$ e que praticavam algum tipo de religião $(82,1 \%)$. Nesse presídio o tempo médio de pena encontrado foi de 2,5 anos (BORGES et al., 2018).

No presente estudo, em relação aos níveis de autoestima das entrevistas, foi identificado que a maioria $(56,8 \%)$ apresentou níveis elevados de autoestima. Comparado com outros estudos, foi identificado um levantamento realizado na Grécia com 307 mulheres, sendo 157 em privação de liberdade e 150 em situação de liberdade. Foi feita uma comparação entre níveis de autoestima e agressividade. Constatou-se que as mulheres presas tinham valores mais baixos de autoestima do que as em liberdade e que apresentavam valores mais elevados de agressividade (KALEMI et al., 2019).

Foi identificado, também, em um estudo realizado no Irã com 130 mulheres em privação de liberdade, utilizando a escala de autoestima de Rosenberg e o inventário de ajustamento de Bell, um perfil sociodemográfico de mulheres em sua maioria entre 21 e 40 anos. Nesse contexto nenhuma das mulheres obtiveram valores de autoestima considerados altos. Sendo 15,4\% moderados e 84,6\% baixos. Os dados com significância em relação a valores mais altos de autoestima foram morar na mesma localidade do presídio e o tempo de pena superior a três anos, divergindo do que foi encontrado no presente estudo (TORKAMAN; MIRI; FAROKHZADLAN, 2018).

Identificou-se que entre as mulheres entrevistadas, uma porcentagem elevada tinha vínculo com alguma religião, tendo esse dado relação significante com níveis mais elevados de autoestima. Uma pesquisa no Rio de Janeiro com presos tanto do sexo masculino quanto do sexo feminino mostrou que 80\% tinham alguma religião e praticavam com certa frequência (CONSTANTINO; DE ASSIS; PINTO, 2016).

Já um estudo realizado na França mostrou que 60\% dos presos praticavam alguma religião e que esse envolvimento estava relacionado com um menor risco de suicídio e com uma menor chance de cometer um novo crime no futuro. Embora 40\% dos presos considerassem a religião como uma forma de lidar com o encarceramento, 23\% viam essas práticas como negativa. Assim o aumento da autoestima tem relação com religiosidade e pode ter também outros benefícios na população em situação de cárcere (MANDHOUJ et al, 2014).

Os resultados demonstraram um escore maior de autoestima nas presidiárias que possuíam filhos e recebiam visitas destes, no contexto de privação de liberdade. Esse fato está de acordo com a literatura, que aponta que as mulheres presidiárias são 
coagidas a elaborarem um luto pela perda de sua liberdade, alienação de sua sexualidade e o abandono de familiares e filhos (SILVA, 2013). Sendo a maternidade um fator percebido como componente da identidade feminina, sua ausência pode desencadear estigmas sociais e, consequentemente, distúrbios da saúde mental, como depressão e baixos níveis de autoestima (FACCHIN et al., 2019).

É fato comprovado que o suporte familiar contribui positivamente para níveis elevados de autoestima (CARAWAN; NALAVANY; JENKINS, 2015). Ademais, o abandono de filhos e de parceiros quando em restrição de liberdade podem ser classificados como fatores precipitantes ou agravantes de desordens da saúde mental destas. Assim, nota-se uma elevada incidência nessas mulheres de distúrbios como depressão, ansiedade, de própria natureza física e autoestima (MIGNON, 2016; SCHULTE; BARBARENA, 2014).

Os dados obtidos neste estudo apontaram um maior escore de autoestima em mulheres que realizavam cuidados pessoais no ambiente de cárcere. Maiores níveis de autoconceito estão relacionados com maiores cuidados individuais e menor negligência e inferioridade da mulher em restrição de liberdade (BASÍLIO et al., 2017).

Identificou-se que as entrevistadas que estavam satisfeitas com o seu próprio corpo possuíam níveis mais elevados de autoestima do que as que estavam insatisfeitas. Evidencia-se que a aparência física é um dos fatores componentes da autoestima, demonstrando o impacto negativo da insatisfação corporal na autoestima (BRECHAN; KVALEM, 2015).

O presente estudo evidencia a importância da propiciação, por parte das instituições penitenciárias, de medidas como atividades físicas/esportivas, dietas balanceadas, produtos de higiene e acompanhamento psicológico; para que possam contribuir para uma melhor autoimagem corporal, autocuidados e autoestima, proporcionando uma melhor saúde mental para estas mulheres que se encontram em privação de liberdade, fator que é essencial para sua posterior reinserção na sociedade (AUDI et al., 2016).

\section{Considerações finais}

O trabalho evidenciou que as mulheres em privação de liberdade que foram estudadas apresentam em sua maioria, níveis elevados de autoestima. Os principais 
fatores que se relacionaram a essas taxas de autoestima foram: a prática de alguma religião, receber visitas dos filhos, ter cuidados pessoais, ser vaidosa, se considerar bonita e estar satisfeita com o próprio corpo. Mostrando que esse valor elevado de autoestima está mais relacionado com questões pessoais subjetivas do que objetivas.

A população privada de liberdade é considerada mais vulnerável do que o resto da população e isso é agravado com o fato de que essas mulheres têm um menor acesso à saúde. A autoestima é um conceito correlacionado com a saúde mental, além de influenciar no bem-estar, na capacidade de socialização e na satisfação com a vida. Com esses diversos benefícios observados é inegável a importância de se ter uma autoestima elevada. Dessa forma, é extremamente importante estudar os fatores que interferem com a autoestima dessas presas, de forma a identificar as melhores estratégias para aumentá-la e, consequentemente, melhorar a qualidade de vida dessas mulheres.

Este estudo, apesar de representar uma pequena amostra do cenário brasileiro de mulheres em cárcere, foi capaz de evidenciar a estreita correlação entre a autoestima e questões pessoais da vida das mulheres estudadas. Assim sendo, revela a importância de uma atenção integral à saúde das mulheres em restrição de liberdade.

As entidades prisionais responsáveis devem ser capazes de fornecer não só atendimento médico, mas também apoio psicológico, atividades de lazer e exercício, dieta balanceada, produtos de higiene e visitas familiares. Essas medidas são capazes de integrar os cuidados de saúde às mulheres no âmbito da saúde mental, incrementando sua autoestima, de maneira a favorecer sua posterior reinserção social.

\section{Referências}

ALMEIDA, P. R. C. et al. Condição de saúde de mulheres privadas de liberdade: uma revisão integrativa. Revista Brasileira de Ciências da Saúde, v. 19, n. 1, p. 73-80, 2015.

ANDRADE, E. R.; SOUSA, E. R.; MINAYO, M. C. S. Intervenção visando a auto-estima e qualidade de vida dos policiais civis do Rio de Janeiro. Ciência \& Saúde Coletiva, v. 14, n. 1, p. 275-285, 2009.

AUDI, C. A. F. et al. Inquérito sobre condições de saúde de mulheres encarceradas. Saúde Debate, v. 40, n. 109, p. 112-124, 2016.

AVANCI, J. Q. et al. Adaptação transcultural de escala de auto-estima para adolescentes. Psicologia: Reflexão e Crítica, v. 20, n. 3, p. 397-405, 2007.

BASÍLIO, L. R. M. et al. Self-concept dialectical transformation: a study in a women's prison. Estudos de Psicologia, v. 34, n. 2, p. 305-314, 2017. 
BASTOS, J. L. D.; DUQUIA, R. P. Um dos delineamentos mais empregados em epidemiologia: estudo transversal. Scientia Medica, v. 17, n. 4, p. 229-232, 2007.

BORGES, A. P. et al. Perfil socioeconômico e sexual de mulheres privadas de liberdade. Revista enfermagem UFPE, v. 12, n. 7, p. 1978-1985, 2018.

BRASIL. Ministério da Justiça e da Segurança Pública. Levantamento Nacional de Informações penitenciárias INFOPEN mulheres. Brasília, DF, 2018.

BRASIL. Ministério da Saúde e Ministério da Justiça. Política nacional de atenção integral a saúde das pessoas privadas de liberdade no sistema prisional. Brasília, DF, 2014.

BRASIL. Constituição da República Federativa do Brasil. Brasília: Senado Federal, 1988.

BRECHAN, I.; KVALEM, I. L. Relationship between body dissatisfaction and disordered eating: Mediating role of self-esteem and depression. Eating Behaviors, v.17, n. 1, p. $49-58,2015$.

CARAWAN, L. W.; NALAVANY, B. A.; JENKINS, C. Emotional experience with dyslexia and self-esteem: the protective role of perceived family support in late adulthood. Aging \& Mental Health, v.20, n. 3, p. 284-294, 2015.

CONSTANTINO, P; DE ASSIS, S. G.; PINTO, L. W. O impacto da prisão na saúde mental dos presos do estado do Rio de Janeiro, Brasil. Ciência \& Saúde Coletiva, v. 21, n.7, p. 2089-2099, 2016.

DIAS, M. S. et al. Auto-estima e fatores associados em gestantes da cidade de Pelotas, Rio Grande do Sul, Brasil. Cadernos de Saúde Pública, Rio de Janeiro, v. 24, n. 12, p. 2787-2797, 2008.

DINI, G. M.; QUARESMA, M. R.; FERREIRA, L. M. Adaptação Cultural e Validação da Versão Brasileira da Escala de Auto-estima de Rosenberg. Revista da Sociedade Brasileira de Cirurgia Plástica, v. 19, n. 1, p. 41-52, 2004.

FACCHIN, F. et al. A woman's worth: the psychological impact of beliefs about motherhood, female identity, and infertility on childless women with endometriosis. Journal of Health Psychology, p. 1-9, 2019.

KALEMI, G. et al. Self-esteem and aggression in women: differences between female prisoners and women without criminal records. Women \& Health, v. 5, p. 1-13, 2019.

MAÇOLA, L.; DO VALE, I. N.; CARMONA, E. V. Avaliação da autoestima de gestantes com uso da Escala de Autoestima de Rosenberg. Revista Escola de Enfermagem USP, v. 44, n. 3, p. 570-577, 2009. 
MANDHOUJ, O. et al. Spirituality and Religion Among French Prisoners: An Effective Coping Resource? International Journal of Offender Therapy and Comparative Criminology. v. 58, n.7, p. 821-834, 2014.

MIGNON, S. Health issues of incarcerated women in the United States. Ciência \& Saúde Coletiva, v. 21, n. 7, p. 2051-2059, 2016.

MEURER, S. T. et al. Validade de construto e consistência interna da escala de autoestima de Rosenberg para uma população de idosos brasileiros praticantes de atividades físicas. Motricidade, vol. 8, n. 4, p. 5-15, $2012 \mathrm{a}$.

MEURER, S. T. et al. Associação entre sintomas depressivos, motivação e autoestima de idosos praticantes de exercícios físicos. Revista Brasileira de Ciências do Esporte, v. 34, n. 3, p. 683-695, 2012 b.

ROTHMAN, K. J.; GREENLAND, S.; LASH, T. L. Epidemiologia moderna. 3.ed. Porto Alegre: Artmed, 2011. 887p.

SCHULTE, N. K.; BARBARENA, L. A. V. Ecomoda: responsabilidade socioambiental aplicada a mulheres presidiárias. Cidadania em Ação: Revista de Extensão e Cultura, v. 8, n. 2, p. 46-64, 2014.

SCHULTHEISZ, T. S. V.; APRILE, M. R. Autoestima, conceitos correlatos e avaliação. Revista Equilíbrio Corporal e Saúde, v. 5, n. 1, p. 36-48, 2013.

SILVA, E. M. A Mulher Detenta, a sua Saúde Sexual e a sua Sexualidade: revisão sistemática da literatura brasileira sobre atuação da enfermagem neste processo. Dissertação (Mestrado em Enfermagem) - Escola de Enfermagem de Ribeirão Preto, Universidade de São Paulo, Ribeirão Preto.

SOUZA, G. C.; CABRAL, K. D. S.; SALGUEIRO, C. D. B L. Reflexões sobre a assistência em enfermagem à mulher encarcerada: Um estudo de revisão integrativa. Arquivo de Ciência da Saúde UNIPAR, v. 22, n. 1, p. 55-62, 2018.

TORKAMAN, M.; MIRI, S.; FAROKHZADLAN, J. Relationship between adaptation and self-esteem in addicted female prisoners in the South east of Iran. International Journal of Adolescent Medicine and Health, v. 41, n. 2, p. 138-143, 2018.

VISCARDI, A. A. F.; CORREIA, P. M. S. Questionários de avaliação da autoestima e/ou da autoimagem: vantagens e desvantagens na utilização com idosos. Revista Brasileira de Qualidade de Vida, v. 9, n. 3, p. 261-280, 2017. 